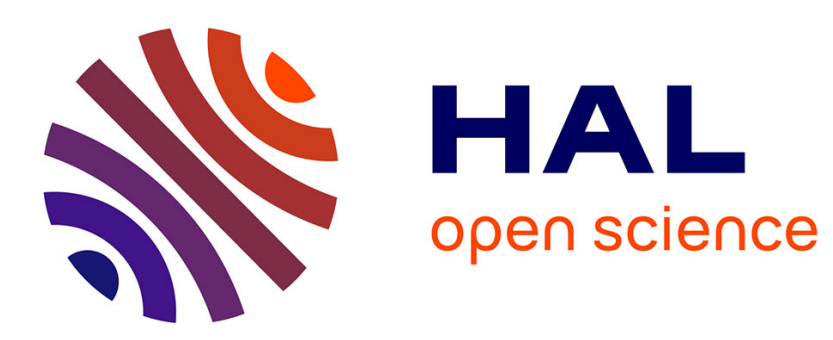

\title{
Some remarks on spanning families and weights for high order Whitney spaces on simplices
}

Ana Alonso Rodríguez, Francesca Rapetti

\section{To cite this version:}

Ana Alonso Rodríguez, Francesca Rapetti. Some remarks on spanning families and weights for high order Whitney spaces on simplices. Computers \& Mathematics with Applications, 2019, 78 (9), pp.2961-2972. 10.1016/j.camwa.2019.03.006 . hal-02411192

\section{HAL Id: hal-02411192 \\ https://hal.science/hal-02411192}

Submitted on 16 Dec 2019

HAL is a multi-disciplinary open access archive for the deposit and dissemination of scientific research documents, whether they are published or not. The documents may come from teaching and research institutions in France or abroad, or from public or private research centers.
L'archive ouverte pluridisciplinaire $\mathbf{H A L}$, est destinée au dépôt et à la diffusion de documents scientifiques de niveau recherche, publiés ou non, émanant des établissements d'enseignement et de recherche français ou étrangers, des laboratoires publics ou privés. 


\title{
Some remarks on spanning families and weights for high order Whitney spaces on simplices
}

\author{
Ana Alonso Rodríguez*, Francesca Rapetti ${ }^{\circ}$ \\ * Dip. di Matematica, Univ. degli Studi di Trento, Povo, Trento, Italy. \\ ana.alonso@unitn.it \\ ${ }^{\circ}$ Dep. de Mathématiques, Univ. Côte d'Azur, Parc Valrose, Nice, France. \\ francesca.rapetti@unice.fr
}

\begin{abstract}
High order Whitney finite element spaces generally lack natural choices of bases but they do have spanning families. In these pages, we recall such a family on simplicial meshes and we prove theoretically its effectiveness. We also comment on some aspects of a new set of degrees of freedom, the so-called weights on the small simplices, to represent discrete functions in these spaces.
\end{abstract}

Keywords: High order Whitney forms on simplices, spanning family, field reconstruction, new degrees of freedom 2000 MSC: 65M60, 74J05

\section{Introduction}

Widely used high order finite element spaces, such as those considered in [10], for the discretization of electromagnetic problems on simplicial meshes are spaces of trimmed polynomial $k$-forms of degree $r+1$ on simplices, with $r \geq 0$, generally denoted $\mathcal{P}_{r+1}^{-} \Lambda^{k}$ in the recent literature. These spaces, known as discrete equivalents of minimal dimension of the $H$ (curl), if $k=1$, or of the $H$ (div), if $k=2$, functional spaces, generally lack natural choices of bases but they do have spanning families (see [9], [3], [13] or the more recent work [1]). The problem of defining spanning families for finite element space fitting some requirements is at the heart of the up-to-date literature. See for example [15], where a partially orthonormal basis with better conditioning properties is proposed for simplicial edge elements, or [6] for finite element complexes similar to those described in [10] with enhanced continuity properties.

In this work, we wish to show how one can compute with one of these spanning families. We focus on the general methodology to follow in the high order framework rather than on the particular PDE or function in exam and we prove theoretical results on its effectiveness. Indeed, the approximation of a PDE problem in $\mathcal{P}_{r+1}^{-} \Lambda^{k}$ or the reconstruction of a discrete function $u_{h} \in$ $\mathcal{P}_{r+1}^{-} \Lambda^{k}$ from a given set of degrees of freedom, lead to a linear system to solve which has the same structure in both cases. 
We consider the spanning family introduced in [13] together with a new set of degrees of freedom to represent functions belonging to the spaces $\mathcal{P}_{r+1}^{-} \Lambda^{k}$, with $r>0$ and $k=0, \ldots, 3$. Both the definitions of the family and of the new degrees of freedom are associated in a very natural way with a collection of particular $k$-simplices, the so-called small $k$-simplices, defined in the mesh. In each mesh simplex, the spanning family for degree $r+1$ include all possible products between the Whitney basis $k$-forms of polynomial degree 1 and homogeneous polynomials of degree $r$ in the barycentric coordinates of the simplex. The new degrees of freedom are integrals (weights) of a $k$-form $u_{h}$ over these particular small $k$-simplices. Note that, for $r=0$, the weights coincide with the classical moments for $u_{h} \in \mathcal{P}_{r+1}^{-} \Lambda^{k}$ as defined in [10], since the small $k$-simplices concide with the $k$-simplices of the mesh, and the spanning family reduces to the well-known basis of Whitney $k$-forms of polynomial degree 1 .

The advantage of the considered spanning family is the simplicity of its explicit definition and for the weights on small $k$-simplices is that, for functions in $\mathcal{P}_{r+1}^{-} \Lambda^{k}$, they all are integrals over $k$-chains, so their physical interpretation (as circulations, fluxes, etc.) is straightforward. In [7], these particular degrees of freedom have been proved to be unisolvent in $\mathcal{P}_{r+1}^{-} \Lambda^{k}$. However, for $r>0$ and $k=1,2$, the number of small $k$-simplices is greater than the dimension of the space $\mathcal{P}_{r+1}^{-} \Lambda^{k}$. It can indeed be proved that the elements of the spanning family satisfy some linear relations and some of the weights are redundant.

Here we investigate how to represent efficiently a trimmed polynomial $k$ form, $u_{h}$, of degree $r+1$ starting from these degrees of freedom. Two different approaches are possible for $r>0$ : either we keep all the weights or we eliminate the redundancies by making a suitable selection of the weights. For the first approach the starting point is the choice of the particular set of generators with cardinality equal to the number of weights and the relationships between its elements given in [13], Proposition 3.5. We thus impose some constraints, to fix a unique representation of $u_{h}$ in terms of this set of generators, by means of Lagrange multipliers getting a non symmetric square linear system to solve. We will see that the same constraints are imposed even while seeking for the approximated solution $u_{h}$ of a PDE problem that needs to be solved in the space $\mathcal{P}_{r+1}^{-} \Lambda^{k}$ with this set of generators. For the second approach the key point is to select a basis of $\mathcal{P}_{r+1}^{-} \Lambda^{k}$ from the proposed set of generators and to choose an unisolvent subset of weights that allow for the determination of $u_{h}$ by solving an invertible linear system whose matrix is a generalized Vandermonde one.

\section{Notations}

Let $\mathcal{P}_{r}\left(\mathbb{R}^{d}\right)$ and $\mathcal{H}_{r}\left(\mathbb{R}^{d}\right)$ be, respectively, the space of polynomials in $d$ variables of degree at most $r$ and the space of homogenous polynomials in $d$ variables of degree equal to $r$. Then, $\mathcal{P}_{r} \Lambda^{k}\left(\mathbb{R}^{d}\right)=\mathcal{P}_{r} \Lambda^{k}$ and $\mathcal{H}_{r} \Lambda^{k}\left(\mathbb{R}^{d}\right)=\mathcal{H}_{r} \Lambda^{k}$ denote the corresponding spaces of polynomial differential forms. The space of trimmed polynomial differential forms $\mathcal{P}_{r}^{-} \Lambda^{k}$ can be defined by involving the Koszul operator, $\kappa: \Lambda^{k+1} \rightarrow \Lambda^{k}$ being $\Lambda^{k}$ the space of $k$-differential forms in $\mathbb{R}^{d}$ (see, e.g. 
$[2])$, that satisfies

$$
\kappa(u \wedge \eta)=(\kappa u) \wedge \eta+(-1)^{k} u \wedge(\kappa \eta), \quad u \in \Lambda^{k}, \quad \eta \in \Lambda^{l} .
$$

Moreover, $\kappa(f u)=f \kappa u$ if $f$ is a function and $\kappa\left(\mathrm{d} x_{i}\right)=x_{i}$. These properties fully determine $\kappa$ and yield $\kappa\left(\mathrm{d} x_{i} \wedge \mathrm{d} x_{j}\right)=x_{i} \mathrm{~d} x_{j}-x_{j} \mathrm{~d} x_{i}, \kappa\left(\mathrm{d} x_{i} \wedge \mathrm{d} x_{j} \wedge \mathrm{d} x_{k}\right)=$ $x_{i} \mathrm{~d} x_{j} \wedge \mathrm{d} x_{k}-x_{j} \mathrm{~d} x_{i} \wedge \mathrm{d} x_{k}+x_{k} \mathrm{~d} x_{i} \wedge \mathrm{d} x_{j}$. In particular $\kappa: \mathcal{H}_{r} \Lambda^{k+1} \rightarrow \mathcal{H}_{r+1} \Lambda^{k}$ and, for $d=3, \kappa u$ is equal to $\mathbf{x} \cdot \mathbf{u}(k=0), \mathbf{x} \times \mathbf{u}(k=1), \mathbf{x} u(k=2)$, and $0(k=3)$, respectively. The space $\mathcal{P}_{r}^{-} \Lambda^{k}$ is intermediate between $\mathcal{P}_{r-1} \Lambda^{k}$ and $\mathcal{P}_{r} \Lambda^{k}$ as follows:

$$
\mathcal{P}_{r}^{-} \Lambda^{k}=\mathcal{P}_{r-1} \Lambda^{k}+\kappa \mathcal{H}_{r-1} \Lambda^{k+1}=\left\{u \in \mathcal{P}_{r} \Lambda^{k}: \kappa u \in \mathcal{P}_{r} \Lambda^{k-1}\right\} .
$$

Let $\Omega$ be a bounded polyhedral domain of $\mathbb{R}^{d}$ and $\mathcal{T}_{h}$ a simplicial mesh of $\Omega$. The spaces of finite element differential forms with respect to the triangulation $\mathcal{T}_{h}$ are denoted $\mathcal{P}_{r}^{-} \Lambda^{k}\left(\mathcal{T}_{h}\right)$. By $H \Lambda^{k}(\Omega)$ we denote the Sobolev space $H^{1}(\Omega)$ if $k=0, H(\operatorname{curl} ; \Omega)$ if $k=1, H(\operatorname{div} ; \Omega)$ if $k=2$ and the space $L^{2}(\Omega)$ if $k=3$. Then we define

$$
\mathcal{P}_{r}^{-} \Lambda^{k}\left(\mathcal{T}_{h}\right)=\left\{u \in H \Lambda^{k}(\Omega): u_{\mid T} \in \mathcal{P}_{r}^{-} \Lambda^{k} \text { for all } T \in \mathcal{T}_{h}\right\} .
$$

It is well known (see, for instance,[3]) that these finite element spaces are that of the Lagrange finite elements of degree $r$ if $k=0$, the first family of Nédélec finite elements of order $r$ conforming in $H(\operatorname{curl} ; \Omega)$ if $k=1$, the first family of Nédélec finite elements of order $r$ conforming in $H(\operatorname{div} ; \Omega)$ if $k=2$, and discontinuous elements of degree $\leq r-1$ if $k=3$.

For $0 \leq j \leq 3$, let $\Delta_{j}(T)$ be the set of all $j$-dimensional subsimplices of the 3-simplex $T \in \mathcal{T}_{h}$ and $\Delta_{j}\left(\mathcal{T}_{h}\right)=\cup_{T \in \mathcal{T}_{h}} \Delta_{j}(T)$. If $\Delta_{0}\left(\mathcal{T}_{h}\right)=\left\{\mathbf{v}_{i}\right\}_{i=1}^{N_{V}}$ then each $k$-simplex $S \in \Delta_{k}\left(\mathcal{T}_{h}\right)$ has associated an increasing map

$$
m_{S}:\{0, \ldots, k\} \rightarrow\left\{1, \ldots, N_{V}\right\} .
$$

This map induces an orientation on $S$. We use the same notation $S$ to denote the oriented $k$-simplex $S=\left[\mathbf{v}_{m_{S}(0)}, \ldots, \mathbf{v}_{m_{S}(k)}\right]$.

If $S \in \Delta_{k}(T)$ then there exists a unique increasing map $m_{S}^{T}:\{0, \ldots, k\} \rightarrow$ $\{0,1,2,3\}$ such that, for each $i \in\{0, \ldots, k\}, m_{S}(i)=m_{T}\left(m_{S}^{T}(i)\right)$.

If $\sigma$ is a $\ell$-subsimplex of the $k$-simplex $S$, with $0 \leq \ell \leq k-1$, we denote by $S-\sigma$ the oriented $(k-1-\ell)$-subsimplex of $S$ with all the vertices in $S$ that are not in $\sigma$. The boundary of $k$-simplex $S$ is the $(k-1)$-chain

$$
\begin{aligned}
\partial_{k}\left[\mathbf{v}_{m_{S}(0)}, \ldots, \mathbf{v}_{m_{S}(k)}\right] & =\sum_{j=0}^{k}(-1)^{j}\left[\mathbf{v}_{m_{S}(0)}, \ldots, \widehat{\mathbf{v}_{m_{S}(j)}}, \ldots, \mathbf{v}_{m_{S}(k)}\right] \\
= & \sum_{j=0}^{k}(-1)^{j}\left(S-\left[\mathbf{v}_{m_{S}(j)}\right]\right) .
\end{aligned}
$$


Let $T$ be a 3 -simplex of $\mathcal{T}_{h}$. For $j \in\{0,1,2,3\}$ we denote $\lambda_{T, j}: T \rightarrow \mathbb{R}$ the baricentric coordinate with respect to $\mathbf{v}_{m_{T}(j)}$. For each $i \in\left\{1, \ldots, N_{V}\right\}$ we denote $\lambda_{i}: \bar{\Omega} \rightarrow \mathbb{R}$

$$
\lambda_{i}(\mathbf{x})=\left\{\begin{array}{cl}
\lambda_{T, j}(\mathbf{x}) & \text { if } \mathbf{x} \in T \text { and } i=m_{T}(j) \text { for some } j \in\{0,1,2,3\} \\
0 & \text { otherwise }
\end{array}\right.
$$

Given $S \in \Delta_{k}\left(\mathcal{T}_{h}\right), 0 \leq k \leq 3$, we denote $w_{S}$ the $k$-form defined in the following way:

- if $k=0$, then $S=\left[\mathbf{v}_{i}\right]$ and $w_{S}=\lambda_{i}$,

- if $k>0$, then $S=\left[\mathbf{v}_{m_{S}(0)}, \ldots, \mathbf{v}_{m_{S}(k)}\right]$ and

$$
w_{S}=\sum_{j=0}^{k}(-1)^{j} \lambda_{m_{S(j)}} \mathrm{d} w_{S-\left[\mathbf{v}_{m_{S}(j)}\right]},
$$

being $\mathrm{d}: \mathcal{P}_{r+1} \Lambda^{k} \rightarrow \mathcal{P}_{r} \Lambda^{k+1}$ the exterior derivative operator.

It is well known that $\mathcal{P}_{1}^{-} \Lambda^{k}\left(\mathcal{T}_{h}\right)=\operatorname{Span}\left\{w_{S}: S \in \Delta_{k}\left(\mathcal{T}_{h}\right)\right\}$.

For $\ell, n \in \mathbb{N}$ let us set

$$
\mathcal{I}(\ell, n+1)=\left\{\boldsymbol{\alpha}=\left(\alpha_{0}, \ldots \alpha_{n}\right) \in \mathbb{N}^{n+1}: \sum_{i=0}^{n} \alpha_{i}=\ell\right\} .
$$

It is easy to check that $\operatorname{card}(\mathcal{I}(\ell, n+1))=\left({ }^{n+\ell} \ell\right)$.

In the sequel we will use the set of pairs

$$
\mathcal{J}_{r, k}(T):=\left\{(\boldsymbol{\alpha}, S): \boldsymbol{\alpha} \in \mathcal{I}(r, 4), S \in \Delta_{k}(T)\right\}
$$

whose cardinality is card $\mathcal{J}_{r, k}(T)=\left({ }^{3+r}{ }_{r}\right)\left({ }^{4}{ }_{k+1}\right)$

\section{Generators of $\mathcal{P}_{r+1}^{-} \Lambda^{k}(T)$ and $\mathcal{P}_{r+1}^{-} \Lambda^{k}\left(\mathcal{T}_{h}\right)$}

Let $T$ be a 3 -simplex of $\mathcal{T}_{h}$. Given $\boldsymbol{\alpha} \in \mathcal{I}(r, 4)$ we denote $\lambda_{T}^{\boldsymbol{\alpha}}=\Pi_{j=0}^{3} \lambda_{m_{T}(j)}^{\alpha_{j}}$. To each pair $(\boldsymbol{\alpha}, S) \in \mathcal{J}_{r, k}(T)$ we associate the $k$-form

$$
\xi_{(\boldsymbol{\alpha}, S)}=\lambda_{T}^{\boldsymbol{\alpha}} w_{S} \in \mathcal{P}_{r+1}^{-} \Lambda^{k}(T) .
$$

It is known (see, e.g. [13]) that

$$
\mathcal{P}_{r+1}^{-} \Lambda^{k}(T)=\operatorname{Span}\left\{\xi_{(\boldsymbol{\alpha}, S)}=\lambda_{T}^{\boldsymbol{\alpha}} w_{S}: \boldsymbol{\alpha} \in \mathcal{I}(r, 4), S \in \Delta_{k}(T)\right\} .
$$

However (see e.g. [7])

$$
N_{r, k}=\operatorname{dim}\left(\mathcal{P}_{r+1}^{-} \Lambda^{k}(T)\right)=\left(\begin{array}{c}
r+k \\
k
\end{array}\right)\left(\begin{array}{c}
4+r \\
3-k
\end{array}\right)
$$




$$
\leq\left(\begin{array}{c}
r+3 \\
r
\end{array}\right)\left(\begin{array}{c}
4 \\
k+1
\end{array}\right)=N_{r, k}^{\prime}=\operatorname{card} \mathcal{J}_{r, k}(T),
$$

and the equality holds only if $k=3$. Hence, if $k=0,1,2$ the set

$$
\mathcal{G}_{r, k}^{\prime}(T)=\left\{\xi_{(\boldsymbol{\alpha}, S)}=\lambda_{T}^{\boldsymbol{\alpha}} w_{S}: \boldsymbol{\alpha} \in \mathcal{I}(r, 4), S \in \Delta_{k}(T)\right\}
$$

generates $\mathcal{P}_{r+1}^{-} \Lambda^{k}(T)$ but it is not a basis.

Remark 1. In [3] it is proved that the subset

$\mathcal{G}_{r, k}(T)=\left\{\xi_{(\boldsymbol{\alpha}, S)}=\lambda_{T}^{\boldsymbol{\alpha}} w_{S}: \boldsymbol{\alpha} \in \mathcal{I}(r, 4), S \in \Delta_{k}(T)\right.$ and $\alpha_{i}=0$ if $\left.i<m_{S}^{T}(0)\right\}$ is a basis of $\mathcal{P}_{r+1}^{-} \Lambda^{k}(T)$.

In the following we investigate how to use in an efficient way the whole set of generators $\mathcal{G}_{r, k}^{\prime}(T)$. It will be convenient to order the set of pairs $\mathcal{J}_{r, k}(T):=$ $\left\{(\boldsymbol{\alpha}, S): \boldsymbol{\alpha} \in \mathcal{I}(r, 4), S \in \Delta_{k}(T)\right\}=\left\{(\boldsymbol{\alpha}, S)_{\ell}: \ell=1, \ldots, N_{r, k}^{\prime}\right\}$ and to use the index $\ell \in\left\{1,2, \ldots, N_{r, k}^{\prime}\right\}$ to denote the functions $\xi_{(\boldsymbol{\alpha}, S)}$, namely, $\xi_{\ell}$ the function corresponding to the $\ell$-pair $(\boldsymbol{\alpha}, S)_{\ell}$.

The key point will be to identify $N_{r, k}^{\prime}-N_{r, k}$ linear combinations of elements of $\mathcal{G}_{r, k}^{\prime}(T)$, namely, coefficients $b_{n, l}$ with $n=1, \ldots, N_{r, k}^{\prime}-N_{r, k}$ and $l=1, \ldots, N_{r, k}^{\prime}$ such that

$$
\sum_{l=1}^{N_{r, k}^{\prime}} b_{n, l} \xi_{l}=0 \text { for } n=1, \ldots, N_{r, k}^{\prime}
$$

and such that the matrix $B_{T}$ with entries $b_{n, l}$ is full rank. Let su recall the following result that is Proposition 3.5 in [13]. (It is also Proposition 3.3 in [7].)

Proposition 1. For $k=0,1,2$ if $S$ is a $(k+1)$-subsimplex of $T$ then

$$
\sum_{i=0}^{k+1}(-1)^{i} \lambda_{m_{S}(i)} w_{S-\left[\mathbf{v}_{m_{S}(i)}\right]}=0 .
$$

Clearly we have also

$$
\lambda_{T}^{\beta} \sum_{i=0}^{k+1}(-1)^{i} \lambda_{m_{S}(i)} w_{S-\left[\mathbf{v}_{m_{S}(i)}\right]}=\sum_{i=0}^{k+1}(-1)^{i} \lambda_{T}^{\boldsymbol{\beta}+\mathbf{e}_{m_{S}^{T}(i)}} w_{S-\left[\mathbf{v}_{m_{S}(i)}\right]}=0
$$

for all $\boldsymbol{\beta} \in \mathcal{I}(r-1,4)$ and $S \in \Delta_{k+1}(T)$. These are $\left({ }^{r+2}{ }_{3}\right)\left({ }^{4}{ }_{k+2}\right)$ equations.

However, in general, these equations are not independent. We now detail an example.

Example 1: Let us consider $T=\left[\mathbf{v}_{0}, \mathbf{v}_{1}, \mathbf{v}_{2}, \mathbf{v}_{3}\right], k=1$ and $r>2$. If $\beta_{0} \neq 0$ then $\boldsymbol{\beta}=\boldsymbol{\gamma}+\mathbf{e}_{0}$ for some $\boldsymbol{\gamma} \in I(r-2,4)$. For $S=\left[\mathbf{v}_{1}, \mathbf{v}_{2}, \mathbf{v}_{3}\right]$, the equation

$$
0=\lambda_{T}^{\beta} \sum_{i=0}^{k+1}(-1)^{i} \lambda_{m_{S}(i)} w_{S-\left[\mathbf{v}_{m_{S}(i)}\right]}=\lambda_{T}^{\gamma} \lambda_{0} \sum_{i=0}^{k+1}(-1)^{i} \lambda_{i+1} w_{S-\left[\mathbf{v}_{i+1}\right]},
$$


is a consequence of the equations associated to the other three faces of the tetrahedra.

Denoting $w_{\left[j j^{\prime}\right]}=w_{\left[\mathbf{v}_{j}, \mathbf{v}_{j^{\prime}}\right]}$, it is easy to check that

$$
\begin{aligned}
\lambda^{\gamma} \lambda_{0}\left[\lambda_{1} w_{[23]}-\lambda_{2} w_{[13]}+\lambda_{3} w_{[12]}\right] & =\lambda^{\gamma} \lambda_{3}\left[\lambda_{0} w_{[12]}-\lambda_{1} w_{[02]}+\lambda_{2} w_{[01]}\right] \\
& -\lambda^{\gamma} \lambda_{2}\left[\lambda_{0} w_{[13]}-\lambda_{1} w_{[03]}+\lambda_{3} w_{[01]}\right] \\
& +\lambda^{\gamma} \lambda_{1}\left[\lambda_{0} w_{[23]}-\lambda_{2} w_{[03]}+\lambda_{3} w_{[02]}\right]
\end{aligned}
$$

In fact $N_{k, r}-N_{k, r}^{\prime}=\left({ }^{r+k}{ }_{k+1}\right)\left({ }^{3+r}{ }_{2-k}\right)$ that for $k=0,1,2$ is smaller than $\left({ }^{r+2}{ }_{3}\right)\left({ }_{k+2}^{4}\right)$. The set of equations to be considered is

$$
\lambda_{T}^{\boldsymbol{\beta}} \sum_{i=0}^{k+1}(-1)^{i} \lambda_{m_{S}(i)} w_{S-\left[\mathbf{v}_{m_{S}(i)}\right]}=0 .
$$

for all $S \in \Delta_{k+1}(T)$ and all $\boldsymbol{\beta} \in \mathcal{I}(r-1,4)$ such that $\beta_{j}=0$ if $j<m_{S}^{T}(0)$.

Proposition 2 (Case $k=2$ ). Let $\boldsymbol{\beta}_{n}$ be the multi-index corresponding to element number $n$ in the set $\mathcal{I}(r-1,4)$ ordered, for instance, lexicographically and for $\ell \in\left\{1, \ldots, N_{r, 2}^{\prime}\right\}$ we consider the couple $(\boldsymbol{\alpha}, S)_{\ell} \in \mathcal{J}_{r, 2}(T)$. We denote $B_{T}$ the matrix with entries

$$
\left(B_{T}\right)_{n, \ell}= \begin{cases}(-1)^{j} & \text { if }(\boldsymbol{\alpha}, S)_{\ell}=\left(\boldsymbol{\beta}_{n}+\mathbf{e}_{i}, T-\left[\mathbf{v}_{m_{T}(i)}\right]\right) \\ 0 & \text { otherwise, }\end{cases}
$$

being $\boldsymbol{\beta}_{n}+\mathbf{e}_{i} \in \mathcal{I}(r, 4)$ the multiindex with components $\left(\boldsymbol{\beta}_{n}+\mathbf{e}_{i}\right)_{\ell}=\left(\boldsymbol{\beta}_{n}\right)_{\ell}+\delta_{i, \ell}$. Then the matrix $B_{T}$ is full rank.

Proof. The number of rows of $B_{T}$ is

$$
\operatorname{card} \mathcal{I}(r-1,4)=\left(\begin{array}{c}
r-1+3 \\
3
\end{array}\right)=N_{r, 2}^{\prime}-N_{r, 2} .
$$

Each row of $B_{T}$ has four elements different from zero, one for each face $T$ $\left[\mathbf{v}_{m_{T}(i)}\right], i=0,1,2,3$, of $T$. The columns of $B_{T}$, that correspond to a couple $(\boldsymbol{\alpha}, S)_{\ell}$ with $S=T-\left[\mathbf{v}_{m_{T}(i)}\right]$ and $(\boldsymbol{\alpha})_{i}=0$ have all the entries equal to zero. Otherwise column $\ell$ has one element different from zero in the row $n$ with $\boldsymbol{\beta}_{n}=\boldsymbol{\alpha}_{\ell}-\mathbf{e}_{i}$. The submatrix obtained diregarding the zero columns has four elements on each row and one element on each column so it is full rank and also $B$ is full rank.

Proposition 3 (Case $k=1)$. For $n \in\left\{1, \ldots,\left({ }^{r-1+3}{ }_{3}\right)\right\}$ let $\boldsymbol{\beta}_{n}$ be the multiindex corresponding to element number $n$ in the set $\mathcal{I}(r-1,4)$ ordered lexicographically. For $\ell \in\left\{1, \ldots, N_{r, 1}^{\prime}\right\}$ we consider the couple $(\boldsymbol{\alpha}, S)_{\ell} \in \mathcal{J}_{r, 1}(T)$. For $j \in\{0,1,2,3\}$ we denote $\Sigma_{j}=T-\left[\mathbf{v}_{m_{T}(j)}\right] \in \Delta_{2}(T)$ and $B_{T, j}$ the matrix with entries

$$
\left(B_{T, j}\right)_{n, \ell}= \begin{cases}(-1)^{i+j} & \text { if }(\boldsymbol{\alpha}, S)_{\ell}=\left(\boldsymbol{\beta}_{n}+\mathbf{e}_{m_{\Sigma_{j}}^{T}(i)}, \Sigma_{j}-\left[\mathbf{v}_{m_{\Sigma_{j}(i)}}\right]\right) \\ 0 & \text { otherwise. }\end{cases}
$$


We denote $\widetilde{B}_{T, 0}$ the submatrix of $B_{T, 0}$ given by its first $\left({ }^{r-1+2}{ }_{2}\right)$ rows.

Then the matrix

$$
B_{T}=\left[\begin{array}{c}
B_{T, 3} \\
B_{T, 2} \\
B_{T, 1} \\
\widetilde{B}_{T, 0}
\end{array}\right] \in \mathbb{N}^{\left(N_{r, 1}^{\prime}-N_{r, 1}\right)} \times N_{r, 1}^{\prime} .
$$

is full rank.

Proof. The number of rows of $B_{T}$ is

$$
\begin{aligned}
3 \operatorname{card} \mathcal{I}(r-1,4)+\operatorname{card} \mathcal{I}(r-1,3) & =3\left(\begin{array}{c}
r-1+3 \\
3
\end{array}\right)+\left(\begin{array}{c}
r-1+2 \\
2
\end{array}\right) \\
& =\left(\begin{array}{c}
r+1 \\
2
\end{array}\right)(3+r)=N_{r, 1}^{\prime}-N_{r, 1} .
\end{aligned}
$$

Let us denote $\widehat{B}_{T}$ the submatrix of $B_{T}$ given by the columns with $S=$ $\left[\mathbf{v}_{m_{T}(1)}, \mathbf{v}_{m_{T}(2)}\right],\left[\mathbf{v}_{m_{T}(1)}, \mathbf{v}_{m_{T}(3)}\right]$ or $\left[\mathbf{v}_{m_{T}(2)}, \mathbf{v}_{m_{T}(3)}\right]$.

Each row of $B_{T}$ has three elements different from zero. The rows of $B_{T, 3}$, $B_{T, 2}$ and $B_{T, 1}$ have one element different from zero in $\widehat{B}_{T}$ while the rows of $\widetilde{B}_{T, 0}$ have the three elements different form zero in $\widehat{B}_{T}$

The columns of $\widehat{B}_{T}$ have exactly one element different from zero because if $\alpha_{0} \neq 0$ then $(\boldsymbol{\alpha}, S)$ has an entry different from zero in $\boldsymbol{\beta}=\boldsymbol{\alpha}-\mathbf{e}_{0}$ and $\Sigma_{j}=\left[v_{m_{T}(0)}, v_{m_{S}(0)}, v_{m_{S}(1)}\right]$ but not in $\widetilde{B}_{T, 0}$ while if $\alpha_{0}=0$ then $(\boldsymbol{\alpha}, S)$ has an entry different from zero in $\widetilde{B}_{T, 0}$. Hence $\widehat{B}_{T}$ is full rank and also $B_{T}$ is full rank.

Now we consider global generators, namely, generators of $\mathcal{P}_{r+1}^{-} \Lambda^{k}\left(\mathcal{T}_{h}\right)$.

\section{Definition 1.}

$\mathcal{G}_{r, k}^{\prime}\left(\mathcal{T}_{h}\right)=\left\{\lambda_{i}^{\alpha_{0}} \lambda_{j}^{\alpha_{1}} \lambda_{\ell}^{\alpha_{2}} \lambda_{n}^{\alpha_{3}} w_{S}: T=\left[\mathbf{v}_{i}, \mathbf{v}_{j}, \mathbf{v}_{\ell}, \mathbf{v}_{n}\right] \in \mathcal{T}_{h}, S \subset T\right.$ and $\left.\boldsymbol{\alpha} \in \mathcal{I}(r, 4)\right\}$

Notice that since the elements of $\mathcal{T}_{h}$ are oriented tetrahedra, from $\left[\mathbf{v}_{i}, \mathbf{v}_{j}, \mathbf{v}_{\ell}, \mathbf{v}_{n}\right] \in$ $\mathcal{T}_{h}$ follows that $1 \leq i<j<\ell<n \leq n_{V}$.

\section{Definition 2.}

$$
\begin{gathered}
\mathcal{G}_{r, 2}\left(\mathcal{T}_{h}\right)=\left\{\lambda_{i}^{\alpha_{0}} \lambda_{j}^{\alpha_{1}} \lambda_{\ell}^{\alpha_{2}} \lambda_{n}^{\alpha_{3}} w_{S}: T=\left[\mathbf{v}_{i}, \mathbf{v}_{j}, \mathbf{v}_{\ell}, \mathbf{v}_{n}\right] \in \mathcal{T}_{h} \quad S \subset T,\right. \\
\left.\boldsymbol{\alpha} \in \mathcal{I}(r, 4) \text { and } \alpha_{0}=0 \text { if } i<m_{S}(0)\right\} \\
\mathcal{G}_{r, 1}\left(\mathcal{T}_{h}\right)=\left\{\lambda_{i}^{\alpha_{0}} \lambda_{j}^{\alpha_{1}} \lambda_{\ell}^{\alpha_{2}} \lambda_{n}^{\alpha_{3}} w_{S}: T=\left[\mathbf{v}_{i}, \mathbf{v}_{j}, \mathbf{v}_{\ell}, \mathbf{v}_{n}\right] \in \mathcal{T}_{h} \quad S \subset T,\right. \\
\left.\boldsymbol{\alpha} \in \mathcal{I}(r, 4) \text { with } \alpha_{0}=0 \text { if } i<m_{S}(0) \text { and } \alpha_{1}=0 \text { if } j<m_{S}(0)\right\}
\end{gathered}
$$

Any element of $\mathcal{G}_{r, k}^{\prime}\left(\mathcal{T}_{h}\right)$ and $\mathcal{G}_{r, k}\left(\mathcal{T}_{h}\right)$ is a function defined in $\bar{\Omega}$. The support of $\lambda_{i}^{\alpha_{0}} \lambda_{j}^{\alpha_{1}} \lambda_{\ell}^{\alpha_{2}} \lambda_{n}^{\alpha_{3}} w_{S}$ is contained in the union of the tetrahedra that contains $S$. If the four components of $\boldsymbol{\alpha}$ are different from zero then its support coincides with the tetrahedra $\left[\mathbf{v}_{i}, \mathbf{v}_{j}, \mathbf{v}_{\ell}, \mathbf{v}_{n}\right]$. 


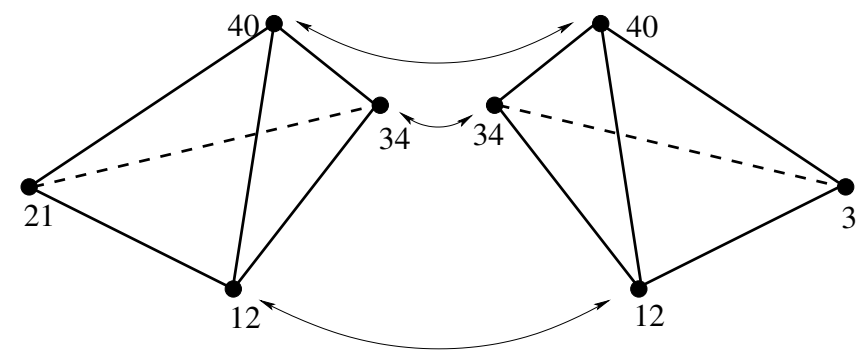

Figure 1: Adjacent tetrahedra $T=[12,21,34,40], T^{\prime}=[3,12,34,40]$, sharing a common face $f=[12,34,40]$.

Both $\mathcal{G}_{r, k}^{\prime}\left(\mathcal{T}_{h}\right)$ and $\mathcal{G}_{r, k}\left(\mathcal{T}_{h}\right)$ generates $\mathcal{P}_{r+1}^{-} \Lambda^{k}\left(\mathcal{T}_{h}\right)$. It is posible to use $\mathcal{G}_{r, k}^{\prime}\left(\mathcal{T}_{h}\right)$ and to impose the relations collected in the matrices $B_{T}$ for all $T \in \mathcal{T}_{h}$ to have a unique representation of the elements of $\mathcal{P}_{r+1}^{-} \Lambda^{k}\left(\mathcal{T}_{h}\right)$. When assembling the matric $B$ that takes into account the relations between the elements of $\mathcal{G}_{r, k}^{\prime}(T)$ for all $T \in \mathcal{T}_{h}$ we notice that some relations hold in the two elements with a common face or, in the case $k=1$, in the elements with an edge in common.

Example 2: Let us assume to work on the simplicial mesh $\mathcal{T}_{h}=\left\{T, T^{\prime}\right\}$ composed of the two tetrahedra $T=\left[\mathbf{v}_{12}, \mathbf{v}_{21}, \mathbf{v}_{34}, \mathbf{v}_{40}\right]$ and $T^{\prime}=\left[\mathbf{v}_{3}, \mathbf{v}_{12}, \mathbf{v}_{34}, \mathbf{v}_{40}\right]$ presented in Figure 1. We set $k=1$ and $r=1$, then we have $N_{1,1}^{\prime}=39$ and $N_{1,1}=32$. Hence matrix $B$ has 7 rows. The relationship

$$
\lambda_{12} w_{\left[\mathbf{v}_{34}, \mathbf{v}_{40}\right]}-\lambda_{34} w_{\left[\mathbf{v}_{12}, \mathbf{v}_{40}\right]}+\lambda_{12} w_{\left[\mathbf{v}_{40}, \mathbf{v}_{34}\right]}=0
$$

holds in the two tetrahedra.

We also observe that the relations in $B_{T, 0}$ that are not in $\widetilde{B}_{T, 0}$ do not appear in any other tetrahedra of the mesh because they involve all the four vertices of a particular tetrahedra.

\section{Solving a PDE over $\mathcal{T}_{h}$}

Let us consider a bilinear form

$$
a(\cdot, \cdot): H \Lambda^{k}(\Omega) \times H \Lambda^{k}(\Omega) \rightarrow \mathbb{R}
$$

symmetric, continuous and coercive on the Sobolev space of differential forms $H \Lambda^{k}(\Omega)$. Given $L \in\left(H \Lambda^{k}(\Omega)^{\prime}\right.$, by the Lax-Milgram lemma, there exists a unique $u \in H \Lambda^{k}(\Omega)$ such that

$$
a(u, v)=L(v) \quad \forall v \in H \Lambda^{k}(\Omega) .
$$

We want to approximate $u$ with a function $u_{h} \in \mathcal{P}_{r+1}^{-} \Lambda^{k}\left(\mathcal{T}_{h}\right)$. Recalling that $\mathcal{P}_{r+1}^{-} \Lambda^{k}\left(\mathcal{T}_{h}\right) \subset H \Lambda^{k}(\Omega)$ for a mesh $\mathcal{T}_{h}$ of $\Omega$, using again the Lax-Milgram lemma, there exists a unique $u_{h} \in \mathcal{P}_{r+1}^{-} \Lambda^{k}\left(\mathcal{T}_{h}\right)$ such that

$$
a\left(u_{h}, v_{h}\right)=L\left(v_{h}\right) \quad \forall v_{h} \in \mathcal{P}_{r+1}^{-} \Lambda^{k}\left(\mathcal{T}_{h}\right) .
$$


The problem now is how to compute $u_{h}$ using the explicit set of generators $\mathcal{G}_{r, k}^{\prime}\left(\mathcal{T}_{h}\right)=\left\{\xi_{\ell}\right\}_{\ell=1}^{N^{\prime}}$ introduced in Section 2. (Notice that $N^{\prime}>N=\operatorname{dim} \mathcal{P}_{r+1}^{-} \Lambda^{k}\left(\mathcal{T}_{h}\right)$ of $\left.\mathcal{P}_{r+1}^{-} \Lambda^{k}\left(\mathcal{T}_{h}\right)\right)$. In particular it holds that

$$
a\left(u_{h}, \xi_{i}\right)=L\left(\xi_{i}\right) \quad \text { for } i=1, \ldots, N^{\prime} .
$$

We consider the matrix $A \in \mathbb{R}^{N^{\prime} \times N^{\prime}}$ and the vector $\mathbf{f} \in \mathbb{R}^{N^{\prime}}$ with entries, resp.,

$$
A_{i, j}=a\left(\xi_{j}, \xi_{i}\right), \quad \mathrm{f}_{i}=L\left(\xi_{i}\right) .
$$

Clearly $A$ is singular but symmetric and positive semidefinite. The linear system $A \mathbf{U}=\mathbf{f}$ has a solution but it si not unique since $\operatorname{Ker} A \neq\{\mathbf{0}\}$. For any solution $\mathbf{U} \in \mathbb{R}^{N^{\prime}}$ of $A \mathbf{U}=\mathbf{f}$, we have $\sum_{j=1} \mathrm{U}_{j} \xi_{j}=u_{h}$, being $u_{h}$ the unique solution of (2).

Proposition 4. The rows of the matrix $B$ are a basis of $\operatorname{Ker} A$.

Proof: We know that for each $\ell=1, \ldots, N^{\prime}-N, \sum_{j=1}^{N^{\prime}} B_{\ell, j} \xi_{j}=0$. Then, for any $i=1, \ldots, N^{\prime}$,

$$
\sum_{j=1}^{N^{\prime}} A_{i, j} B_{\ell, j}=\sum_{j=1}^{N^{\prime}} a\left(\xi_{j}, \xi_{i}\right) B_{\ell, j}=a\left(\sum_{j=1}^{N^{\prime}} B_{\ell, j} \xi_{j}, \xi_{i}\right)=0 .
$$

This means that the rows of $B$ are in $\operatorname{Ker} A$. They are linear independent because $B$ is full rank. Moreover since $\operatorname{Rank} A=N$ then $\operatorname{dim}(\operatorname{Ker} A)=N^{\prime}-N$ so they are a basis $\operatorname{Ker} A$.

Corollary 1. If $\mathbf{f} \in \operatorname{Im} A$ then there exists a unique solution $\mathbf{U} \in \mathbb{R}^{N^{\prime}}$ such that

$$
\begin{aligned}
& A \mathbf{U}=\mathbf{f} \\
& B \mathbf{U}=\mathbf{0} .
\end{aligned}
$$

Proof. It is easy to check that if $A \mathbf{U}=\mathbf{0}$ and $B \mathbf{U}=\mathbf{0}$ then $\mathbf{U}=\mathbf{0}$. In fact from the first equation $\mathbf{U} \in \operatorname{Ker} A$. Since the rows of $B$ are a basis of $\operatorname{Ker} A$, $B \mathbf{U}=\mathbf{0}$ means that $\mathbf{U} \in(\operatorname{Ker} A)^{\perp}$, then $\mathbf{U}=\mathbf{0}$.

If we want to compute $\mathbf{U}$ by solving a square linear system, we have to take into account the following considerations (see [4] for a complete presentation on saddle point problems' treatment).

If a matrix $Q \in \mathbb{R}^{N^{\prime} \times\left(N^{\prime}-N\right)}$ such that its columns are a basis of $\operatorname{Ker}\left(A^{T}\right)=$ $(\operatorname{Im} A)^{\perp}$ is known, then for all $\mathbf{f} \in \mathbb{R}^{N^{\prime}}$ there exists a unique pair $(\mathbf{U}, \boldsymbol{\Lambda}) \in$ $\mathbb{R}^{N^{\prime}} \times \mathbb{R}^{N^{\prime}-N}$ such that

$$
\begin{array}{ll}
A \mathbf{U}+Q \boldsymbol{\Lambda} & =\mathbf{f} \\
B \mathbf{U} & =\mathbf{0} .
\end{array}
$$

In fact, denoting by $\mathbf{q}$ the orthogonal projection of $\mathbf{f}$ in $(\operatorname{Im} A)^{\perp}$, since $\mathbf{f}-\mathbf{q} \in$ $\operatorname{Im} A$, there exists a unique $\mathbf{U} \in \mathbb{R}^{N^{\prime}}$ such that

$$
\begin{aligned}
& A \mathbf{U}=\mathbf{f}-\mathbf{q} \\
& B \mathbf{U}=\mathbf{0} .
\end{aligned}
$$


Since the columns of $Q$ are a basis of $(\operatorname{Im} A)^{\perp}$ and $\mathbf{q} \in(\operatorname{Im} A)^{\perp}$, there exists a unique $\boldsymbol{\Lambda} \in \mathbb{R}^{N^{\prime}-N}$ such that $\mathbf{q}=Q \boldsymbol{\Lambda}$. Clearly, if $\mathbf{f} \in \operatorname{Im} A$ then $\boldsymbol{\Lambda}=\mathbf{0}$. Notice also that $\mathbf{U}$ is the best approximated solution of $A \mathbf{U}=\mathbf{f}$. Finally, it is clear that, if $A$ is symmetric, then $(\operatorname{Im} A)^{\perp}=\operatorname{Ker}\left(A^{T}\right)=\operatorname{Ker}(A)$, so $Q=B^{T}$.

Proposition 5. The matrix

$$
\left[\begin{array}{cc}
A & B^{T} \\
B &
\end{array}\right]
$$

where the missing block is meant to be zero, is invertible.

Proof: This matrix is square so it is enough to prove uniqueness. We will show that if

$$
\begin{array}{ll}
A \mathbf{U}+B^{T} \boldsymbol{\Lambda} & =\mathbf{0} \\
B \mathbf{U} & =\mathbf{0},
\end{array}
$$

then $\mathbf{U}=\mathbf{0}$ and $\boldsymbol{\Lambda}=\mathbf{0}$. Since $B \mathbf{U}=\mathbf{0}$ we have $\mathbf{0}=\mathbf{U}^{T} A \mathbf{U}+\mathbf{U}^{T} B^{T} \boldsymbol{\Lambda}=$ $\mathbf{U}^{T} A \mathbf{U}$. Being $A$ symmetric and positive semidefinite, there exist an orthogonal matrix $P$, which columns are eigenvectors of $A$, and a diagonal matrix $D$ with $D_{i, i}>0$ for $i=1, \ldots, N$ and $D_{i, i}=0$ for $i=N+1, \ldots, N^{\prime}$ such that $P^{T} D P=$ $A$. So we have

$$
0=\mathbf{U}^{T} A \mathbf{U}=\mathbf{U}^{T} P^{T} D P \mathbf{U}=\sum_{i=1}^{N^{\prime}} D_{i, i}(P \mathbf{U})_{i}^{2}=\sum_{i=1}^{N} D_{i, i}(P \mathbf{U})_{i}^{2} .
$$

It follows that $(P \mathbf{U})_{i}=0$ for $i=1, \ldots, N$ hence $D P \mathbf{U}=\mathbf{0}$.. As a consequence $\mathbf{U} \in \operatorname{Ker} A$ because $A \mathbf{U}=P^{T} D P \mathbf{U}=\mathbf{0}$. Since the rows of $B$ are a basis of $\operatorname{Ker} A$ from $B \mathbf{U}=\mathbf{0}$ follows that $\mathbf{U} \in(\operatorname{Ker} A)^{\perp}$ and then $\mathbf{U}=\mathbf{0}$. Finally being, $B$ full rank we have $\operatorname{dim}\left(\operatorname{Ker} B^{T}\right)=0$ and $B^{T} \boldsymbol{\Lambda}=\mathbf{0}$ implies $\boldsymbol{\Lambda}=\mathbf{0}$.

The next result follows from Corollary 1 and Proposition 5.

Corollary 2. For any $\mathbf{f} \in \mathbb{R}^{N^{\prime}}$, the linear system

$$
\begin{array}{ll}
A \mathbf{U}+B^{T} \boldsymbol{\Lambda} & =\mathbf{f} \\
B \mathbf{U} & =\mathbf{0},
\end{array}
$$

has a unique solution. If $\mathbf{f} \in \operatorname{Im} A$, then $\boldsymbol{\Lambda}=\mathbf{0}$.

Remark 2. The meaning of the coefficients $\mathrm{U}_{i}$ used to represent the discrete solution $u_{h}$ in terms of the selected set of generators $\left\{\xi_{i}\right\}_{i=1}^{N^{\prime}}$ is not relevant in the solution procedure. See [11] (resp. [5]) for an application of the proposed approach in the case $k=1$ and $r>0$, where redundancies have been eliminated (resp. have been treated as stated in Corollary 2).

\section{Small simplices and weights}

For $(\boldsymbol{\alpha}, S) \in \mathcal{J}_{r, k}(T)$, we denote a particular $k$-simplex contained in $T$ of the set of the so-called small $k$-simplices, firstly defined in [12]. 

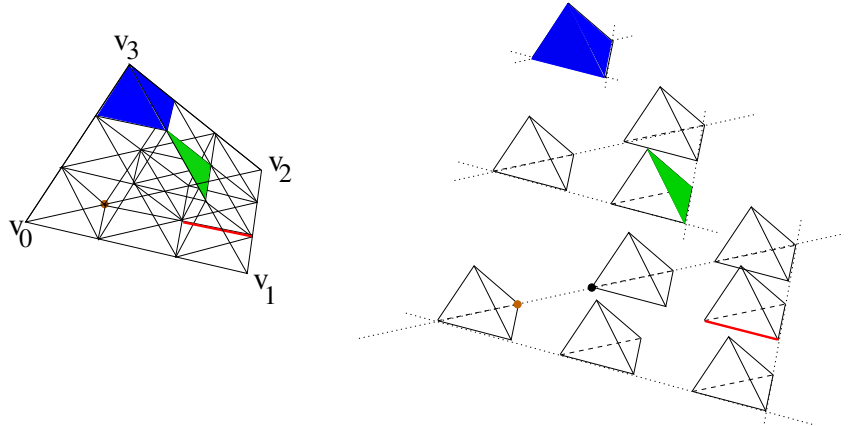

Figure 2: Visualization (on the left) of all the small simplices associated to the principal lattice of degree $r+1=3$ in the tetrahedron $T=\left[\mathbf{v}_{\mathbf{0}} \mathbf{v}_{\mathbf{1}} \mathbf{v}_{\mathbf{2}} \mathbf{\mathbf { v } _ { \mathbf { 3 } }}\right]$. The same set of small simplices in a fragmented visualization (on the right). A small tetrahedron in blue, a small face in green, a small edge in red, and a small nodes in brown (in black, the "twin" node of the brown one).

Let $T=\left[\mathbf{v}_{m_{T}(0)}, \mathbf{v}_{m_{T}(1)}, \mathbf{v}_{m_{T}(2)}, \mathbf{v}_{m_{T}(3)}\right]$ be a 3 -simplex of $\mathcal{T}_{h}$. The principal lattice $L_{r+1}(T)$ of order $r+1$ in the 3 -simplex $T$ is the set

$$
L_{r+1}(T)=\left\{\mathbf{x} \in T: \lambda_{m_{T}(i)}(\mathbf{x}) \in\left\{0, \frac{1}{r+1}, \frac{2}{r+1}, \ldots, \frac{r}{r+1}, 1\right\}, 0 \leq i \leq 3\right\} .
$$

Definition 3. Let $T$ be a 3-simplex of $\mathcal{T}_{h}$ and $\boldsymbol{\alpha} \in \mathcal{I}(r, 4)$.

If $r=0$ and $S \in \Delta_{k}$ (T) for any $k \in\{0,1,2,3\}$ then $\mathbf{s}_{(\boldsymbol{\alpha}, S)}=S$.

If $r>0$ then

- For $k=3$, then $S=T$ and $\mathbf{s}_{(\boldsymbol{\alpha}, S)}$ is the (small) 3-simplex with barycenter at the point of coordinates

$$
\frac{1}{r+1} \sum_{i=0}^{3}\left[\left(\frac{1}{4}+\alpha_{i}\right) \mathbf{v}_{m_{T}(i)}\right],
$$

and $\frac{1}{r+1}$-homothetic to $T$.

- For $k=1,2$ and $S \in \Delta_{k}(T), \mathbf{s}_{(\boldsymbol{\alpha}, S)}$ is the k-subsimplex of $\mathbf{s}_{(\boldsymbol{\alpha}, T)}$ which is parallel to $S$.

- For $k=0$ and $S \in \Delta_{0}(T), \mathbf{s}_{(\boldsymbol{\alpha}, S)}$ is the 0-subsimplex of $\mathbf{s}_{(\boldsymbol{\alpha}, T)}$

$$
\frac{1}{r+1} \sum_{j=0}^{3} \alpha_{j} \mathbf{v}_{m_{T}(j)},
$$

namely the vertex equivalent to $S$ but in $\mathbf{s}_{(\boldsymbol{\alpha}, T)}$.

The orientation of the small simplex $\mathbf{s}_{(\boldsymbol{\alpha}, S)}$ coincides with the one of $S$. See Figure 2 for a presentation of the small $k$-simplices, $k=0, \ldots, d$, in a tetrahedron $T$ for $r+1=3$. 

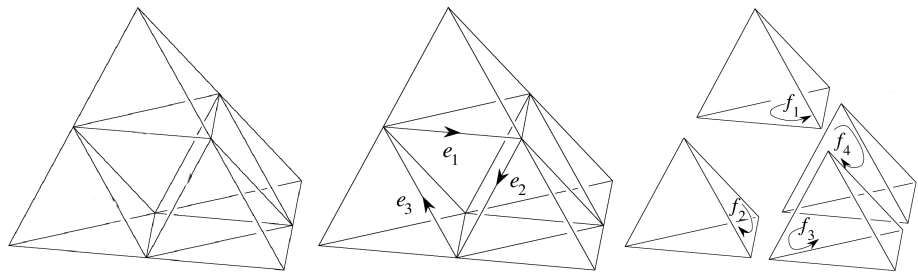

Figure 3: From the principal lattice of degree $r+1=2$ in a tetrahedron $T$, we define a decomposition of $T$ into 4 small tetrahedra, 1 octahedra $O$ and 0 reversed tetrahedra $\perp$ (left). Each face on $\partial T$ is decomposed into 3 small faces and 1 reversed triangle $\nabla$ (center). Each reversed triangle $\nabla$ corresponds to a relation among the 3 generators $\xi_{i}$ of $\mathcal{P}_{2}^{-} \Lambda^{1}$ such that $\mathbf{s}_{i} \in \partial \nabla$ (here $\partial \nabla=\left\{e_{1}, e_{2}, e_{3}\right\}$ ). Each octahedron $O$ corresponds to a relation among the 4 generators $\xi_{j}$ of $\mathcal{P}_{2}^{-} \Lambda^{2}$ such that $\mathbf{s}_{j} \in\left\{f_{1}, f_{2}, f_{3}, f_{4}\right\}$ (right). Note that the 4 faces of the octahedron $O$ which are reversed triangles are not involved in the latter relation.
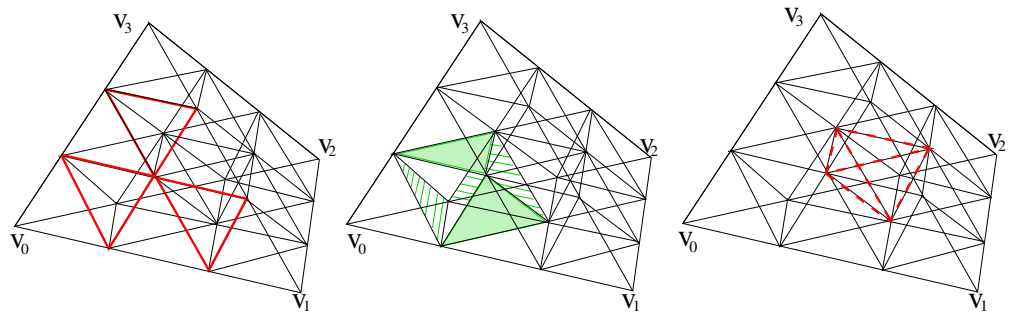

Figure 4: From the principal lattice of degree $r+1=3$ in a tetrahedron $T$, we define a decomposition of $T$ into 10 small tetrahedra, 4 octahedra $O$ and 1 reversed tetrahedron $\perp$. Each face on $\partial T$ is decomposed into 6 small faces and 3 reversed triangle $\nabla$, in solid red line (left). Each reversed triangle $\nabla$ corresponds to a relation among three $\xi_{i}$ of $\mathcal{P}_{3}^{-} \Lambda^{1}$ such that $\mathbf{s}_{i} \in \partial \nabla$. Each octahedron $O$ corresponds to a relation among four $\xi_{j}$ of $\mathcal{P}_{3}^{-} \Lambda^{2}$ such that $\mathbf{s}_{j} \in \partial O$, indicated in green (center). Each of the three out of four faces $\nabla \perp$ of the reversed tetrahedron $\perp$, in dashed red line (right), corresponds to a relation among three $\xi_{i}$ of $\mathcal{P}_{3}^{-} \Lambda^{1}$ such that $\mathbf{s}_{i} \in \partial \nabla \perp$.

Remark 3 (Geometrical interpretation of Proposition 1.). When connecting the points of $L_{r+1}(T)$, for $r>0$, with segments parallel to the edges of $T$ (see Figures 3 and 4 , for $r+1=2$ and $r+1=3$, respectively), we obtain

- a partition of $T$ into exactly $\left({ }^{r+3}{ }_{3}\right)$ small tetrahedra (homothetic to $T$ ), $\left({ }^{r+2}{ }_{3}\right)$ octahedra $O$ and $\left({ }^{r+1}{ }_{3}\right)$ reversed tetrahedra $\perp$;

- a partition of each face $F$ of $\partial T$ into exactly $\left({ }^{r+2}{ }_{2}\right)$ small faces (homothetic to $F)$ and $\left({ }^{r+1}{ }_{2}\right)$ reversed triangles $\nabla$, on $F$.

The resulting octahedra $O$, reversed tetrahedra $\perp$ and reversed triangles $\nabla$ are called "holes" [13]. Each octahedron (resp. each reversed triangle) is in correspondence with a relation stated in Proposition 1 for $k=2($ resp. $k=1)$. This is due to the fact that in Proposition 1 we involve generators of the form $\xi_{\ell}=\lambda^{\boldsymbol{\alpha}} w_{\sigma}$ with $\alpha_{m_{S}^{T}(i)} \neq 0$ and $\sigma=S-\left[v_{m_{S}^{T}(i)}\right]$, and that $\mathbf{s}_{\ell}=\mathbf{s}_{(\boldsymbol{\alpha}, \sigma)}$, with those $\boldsymbol{\alpha}$ and $\sigma$, are small $k$-simplices on the boundary of the "holes". 
The relations for $k=2$ come exclusively from the octahedra. Each octahedron $O$ corresponds to a relation among the four generators $\xi_{j}$ of $\mathcal{P}_{r+1}^{-} \Lambda^{2}$ such that $\mathbf{s}_{j}$ is a small face (see right part of Figure 3 for $r+1=2$ and of Figure 4 for $r+1=3$ ). Therefore, each octahedron $O$ contributes to one relation with four out of the eight faces in $\partial O$ (indeed, four of its faces are reversed triangles, already counted out in the relations for $k=1$ ).

The relations for $k=1$ come exclusively from the reversed triangles, those $\nabla$ contained on each face $F \in \partial T$ and the three $\nabla \perp$ out of four that constitute the boundary of reversed tetrahedra $\perp$ (see center part of Figure 3 for $r+1=2$ and left part of Figure 4 for $r+1=3$ ). More precisely, each reversed triangle $\nabla$ (resp. $\nabla \perp$ ) corresponds to a relation among the three generators $\xi_{i}$ of $\mathcal{P}_{r+1}^{-} \Lambda^{1}$ such that $\mathbf{s}_{i} \in \partial \nabla\left(\right.$ resp. $\left.\mathbf{s}_{i} \in \partial \nabla \perp\right)$.

For $k=0$, Proposition 1 states those relations among generators $\xi_{n}$ of $\mathcal{P}_{r+1}^{-} \Lambda^{0}$ that correspond to say how the (twin) vertices of the small tetrahedra have to be glued to pass from the fragmented configuration (as in Figure 2, right) to the assembled one (as in Figure 2, left).

Now we introduce the notion of weight of $u_{h} \in \mathcal{P}_{r+1}^{-} \Lambda^{k}(T)$ on the small simplex $\mathbf{s}_{(\boldsymbol{\alpha}, S)}$ that we denote $\left\langle u_{h}, \mathbf{s}_{(\boldsymbol{\alpha}, S)}\right\rangle$.

- If $S$ is the 3 -simplex $T$ then $\left\langle u_{h}, \mathbf{s}_{(\boldsymbol{\alpha}, S)}\right\rangle=\int_{\mathbf{s}_{(\boldsymbol{\alpha}, T)}} u_{h}$.

- If $S \in \Delta_{2}(T)$ then $\left\langle u_{h}, \mathbf{s}_{(\boldsymbol{\alpha}, S)}\right\rangle=\int_{\mathbf{s}_{(\boldsymbol{\alpha}, S)}} \mathbf{u}_{h} \cdot \mathbf{n}_{S}$ being

$$
\mathbf{n}_{S}=\frac{\left(\mathbf{v}_{m_{S}(1)}-\mathbf{v}_{m_{S}(0)}\right) \times\left(\mathbf{v}_{m_{S}(2)}-\mathbf{v}_{m_{S}(0)}\right)}{\|\left(\mathbf{v}_{m_{S}(1)}-\mathbf{v}_{m_{S}(0)}\right) \times\left(\mathbf{v}_{m_{S}(2)}-\mathbf{v}_{m_{S}(0)}\right)} .
$$

- If $S \in \Delta_{1}(T)$ then $\left\langle u_{h}, \mathbf{s}_{(\boldsymbol{\alpha}, S)}\right\rangle=\int_{\mathbf{s}_{(\boldsymbol{\alpha}, S)}} \mathbf{u}_{h} \cdot \boldsymbol{\tau}_{S}$ being

$$
\boldsymbol{\tau}_{S}=\frac{\mathbf{v}_{m_{S}(1)}-\mathbf{v}_{m_{S}(0)}}{\left\|\mathbf{v}_{m_{S}(1)}-\mathbf{v}_{m_{S}(0)}\right\|} .
$$

- If $S \in \Delta_{0}(T)$ then $\left\langle u_{h}, \mathbf{s}_{(\boldsymbol{\alpha}, S)}\right\rangle=u_{h}\left(\mathbf{s}_{(\boldsymbol{\alpha}, S)}\right)$.

These small weights are unisolvents in $\mathcal{P}_{r+1}^{-} \Lambda^{k}(T)$, namely, they identify univocally any element of $\mathcal{P}_{r+1}^{-} \Lambda^{k}(T)$. In fact in [7] have been proved the following result.

Proposition 6. Let $T$ be a 3-simplex of $\mathcal{T}_{h}$. If $u_{h} \in \mathcal{P}_{r+1}^{-} \Lambda^{k}(T)$ and

$$
\left\langle u_{h}, \mathbf{s}_{(\boldsymbol{\alpha}, S)}\right\rangle=0
$$

for all $\boldsymbol{\alpha} \in \mathcal{I}(r, 4)$ and $S \in \Delta_{k}(T)$, then $u_{h}=0$.

The small weights are as many as the functions of $\mathcal{G}_{r, k}^{\prime}(T)$. We have ordered the set of pairs $\mathcal{J}_{r, k}(T)=\left\{(\boldsymbol{\alpha}, S)_{\ell}: \ell=1, \ldots, N_{r, k}^{\prime}\right\}$ for each $\ell=1, \ldots, N_{r, k}^{\prime}$ so we denote $\mathbf{s}_{\ell}=\mathbf{s}_{(\boldsymbol{\alpha}, S)_{\ell}}$ (as we do for the elements $\mathcal{G}_{r, k}^{\prime}(T)$ where $\xi_{\ell}=\xi_{(\boldsymbol{\alpha}, S)_{\ell}}$ ). 
Remark 4. As explained above, the functions of $\mathcal{G}_{r, k}^{\prime}(T)$ are in a one-to-one correspondence with the set of pairs $(\boldsymbol{\alpha}, S)$, where the multi-index $\boldsymbol{\alpha}$ is strictly related to a principal lattice defined on $T$, namely a uniform distribution of nodes on $T$, in order to have $\boldsymbol{\alpha}$ with integer components. However, unisolvent $k$-weights can be defined by relying on a set of $k$-chains associated with a nodal distribution on $T$ other than the uniform one used for the multi-indices $\boldsymbol{\alpha}$. In other words, the $k$-chains that constitute the support of the integrals for unisolvent $k$-weights can be associated with a different (thus non-uniform) node distribution in T. But, it will be not possible to know the entries of $B_{T}$ a priori as in the uniform case, since the relations among the elements of $\mathcal{G}_{r, k}^{\prime}(T)$ will be more complicate.

We introduce also the matrix $V \in \mathbb{R}^{N_{r, k}^{\prime} \times N_{r, k}^{\prime}}$ with entries

$$
V_{i, j}=\int_{\mathbf{s}_{i}} \xi_{j}
$$

- If $r=0$ then $V=I$ for $k=0,1,2,3$.

- If $r>0$ and $k=3$ then $V$ is invertible. In fact, it is easy to check that if $V \mathbf{U}=\mathbf{0}$ then $\mathbf{U}=\mathbf{0}$. If $V \mathbf{U}=\mathbf{0}$ then $\int_{\mathbf{s}_{i}} \mathbf{u}_{h}=0$ for all $i=1, \ldots, N_{r, k}^{\prime}$ being $u_{h}=\sum_{j=1}^{N_{r, k}^{\prime}} U_{j} \xi_{j} \in \mathcal{P}_{r+1}^{-} \Lambda^{3}(T)$. Then it follows from proposition 6 that $u_{h}=0$. Since $\left\{\xi_{j}: 1 \leq j \leq N_{r, k}=N_{r, k}^{\prime}\right\}$ is a basis of $\mathcal{P}_{r+1}^{-} \Lambda^{3}(T)$ then it must be $\mathbf{U}=\mathbf{0}$.

- If $r>0$ and $k<3$ the set of functions $\left\{\xi_{j}: j=1, \ldots, N_{r, k}^{\prime}\right\}$ is not linear independent so the matrix $V$ is singular (but $k=0$ is well-known).

Proposition 7. If $r>0$ and $k<3$ then $\operatorname{rank} V=N_{r, k}<N_{r, k}^{\prime}$.

Proof. First we show that the first $N_{r, k}$ columns of $V$ are linear independent. Given $\mathbf{U} \in \mathbb{R}^{N_{r, k}^{\prime}}$ with $U_{j}=0$ for $j=N_{r, k}+1, \ldots, N_{r, k}^{\prime}$ and $V \mathbf{U}=\mathbf{0}$ then $\int_{\mathbf{s}_{i}} \mathbf{u}_{h}=0$ for all $i=1, \ldots, N_{r, k}^{\prime}$ being $u_{h}=\sum_{j=1}^{N_{r, k}} U_{j} \xi_{j} \in \mathcal{P}_{r+1}^{-} \Lambda^{3}(T)$. Then it follows from proposition 6 that $u_{h}=0$. Since $\left\{\xi_{j}: 1 \leq j \leq N_{r, k}\right\}$ is a basis of $\mathcal{P}_{r+1}^{-} \Lambda^{3}(T)$ then it must be $\mathbf{U}=\mathbf{0}$.

Now we show that there are not $N_{r, k}+1$ columns of $V$ linear independent. If we choose $N_{r, k}+1$ different columns of $V, \mathbf{v}_{j(1)} \ldots, \mathbf{v}_{j\left(N_{r, k}+1\right)}$ and we consider the corresponding functions $\xi_{j(1)}, \ldots, \xi_{j\left(N_{r, k}+1\right)}$ since they are not linear independent then we can writte

$$
\xi_{j\left(N_{r, k}+1\right)}=\sum_{k=1}^{N_{r, k}} a_{k} \xi_{j(k)} .
$$

with $\mathbf{a} \in \mathbb{R}^{N_{r, k}} \backslash \mathbf{0}$. So for all $i=1, \ldots, N_{r, k}^{\prime}$

$$
\left[\mathbf{v}_{j\left(N_{r, k}+1\right)}\right]_{i}=\int_{\mathbf{s}_{i}} \xi_{j\left(N_{r, k}+1\right)}=\int_{\mathbf{s}_{i}} \sum_{k=1}^{N_{r, k}} a_{k} \xi_{j(k)}=\sum_{k=1}^{N_{r, k}} a_{k} \int_{\mathbf{s}_{i}} \xi_{j(k)}=\left[\sum_{k=1}^{N_{r, k}} a_{k} \mathbf{v}_{j(k)}\right]_{i} .
$$


This means that the vector $\mathbf{v}_{j\left(N_{r, k}+1\right)}$ is linearly dependent on the vectors $\left\{\mathbf{v}_{j(1)} \ldots, \mathbf{v}_{j\left(N_{r, k}\right)}\right\}$.

We thus have $\operatorname{dim}(\operatorname{Ker} V)=N_{r, k}^{\prime}-N_{r, k}$, since by the rank theorem the following identity, $\operatorname{rank}(V)+\operatorname{dim}(\operatorname{Ker} V)=N_{r, k}^{\prime}$, holds.

The global matrix $V_{g}$ of size $N^{\prime} \times N^{\prime}$ is block-diagonal with all the blocks identical (up to a renumerotation of the local dofs) to the matrix $V$ in one tetrahedron. As it occurred for $B$, the assembling of the global matrix $V_{g}$ starting from the local ones is not done by summing up the contributions of two adjacent tetrahedra, say $T$ and $T^{\prime}$, but just over-writing those entries which appear twice, once from $T$ and once from $T^{\prime}$. The entries $V_{i, j}$ of the local matrix $V$ for one tetrahedron $T$, can be computed numerically either by relying on suitable quadrature formulas [14] or by involving a geometrical interpretation of the weights $\int_{\mathbf{s}_{i}} \xi_{j}$. Indeed, in [7] we proved that (here $\mathbf{s}_{i}=\mathbf{s}_{\left(\boldsymbol{\alpha}^{\prime}, S^{\prime}\right)}$ and $\left.\xi_{j}=\lambda^{\alpha} w_{S}\right)$

$$
\int_{\mathbf{s}_{\left(\boldsymbol{\alpha}^{\prime}, S^{\prime}\right)}} \lambda^{\boldsymbol{\alpha}} w_{S}=\int_{\mathbf{s}_{\left(\boldsymbol{\alpha}^{\prime}, S^{\prime}\right)}} w_{S} \int_{\mathbf{s}_{\left(\boldsymbol{\alpha}^{\prime}, S^{\prime}\right)}} \lambda^{\boldsymbol{\alpha}} /\left|\mathbf{s}_{\left(\boldsymbol{\alpha}^{\prime}, S^{\prime}\right)}\right| .
$$

For the integral of $\lambda^{\boldsymbol{\alpha}}$ on the small $k$-simplex $\mathbf{s}_{\left(\boldsymbol{\alpha}^{\prime}, S^{\prime}\right)}$, we have that

$$
\int_{\mathbf{s}_{\left(\boldsymbol{\alpha}^{\prime}, S^{\prime}\right)}} \lambda^{\boldsymbol{\alpha}}=\frac{\alpha_{0} ! \ldots \alpha_{d} ! k !}{(r+k) !}\left|\mathbf{s}_{\left(\boldsymbol{\alpha}^{\prime}, S^{\prime}\right)}\right|
$$

with $\alpha_{i}$ ! = 1 for all $\alpha_{i}=0$ (this formula is known in the finite element context and its proof can be written by adapting, for example, that of Proposition 3.5 in [11]) and

$$
\int_{\mathbf{s}_{\left(\boldsymbol{\alpha}^{\prime}, S^{\prime}\right)}} w_{S}= \pm\left|(T-S) \vee \mathbf{s}_{\left(\boldsymbol{\alpha}^{\prime}, S^{\prime}\right)}\right| /|T|,
$$

where $\left|(T-S) \vee \mathbf{s}_{\left(\boldsymbol{\alpha}^{\prime}, S^{\prime}\right)}\right|$ is the volume of the tetrahedron contained in $T$ with vertices those of $T-S$ and those of $\mathbf{s}_{\left(\boldsymbol{\alpha}^{\prime}, S^{\prime}\right)}$. The sign depends on whether the orientation on this new tetrahedron coincides or not with that of $T$. The volume of the $d$-simplex $(T \backslash S) \vee \mathbf{s}_{\left(\boldsymbol{\alpha}^{\prime}, S^{\prime}\right)}$ can be computed by a particular determinant.

Example 3: Let us consider the 3-simplex $T=\left[v_{0}, v_{1}, v_{2}, v_{3}\right]$ presented in Figure 5. To treat the case $k=1, r=1$, we introduce the mid-point $v_{i j}$ of the edge $\left[v_{i}, v_{j}\right]$, for $i, j=0, \ldots, 3$. In red, we have indicated two particular tetrahedra, namely $T_{1}=\left[v_{0}, v_{13}, v_{23}, v_{3}\right]$ (left) and $T_{2}=\left[v_{0}, v_{1}, v_{12}, v_{3}\right]$ (right). The thick line denotes the small edge $\mathbf{s}_{\left(\boldsymbol{\alpha},\left[v_{1}, v_{2}\right]\right)}$ with $\boldsymbol{\alpha}^{\prime}$ equal either to $(0,0,0,1)$ (left) or to $(0,1,0,0)$ (right). So

$$
\int_{\mathbf{s}_{\left((0,0,0,1),\left[v_{1}, v_{2}\right]\right)}} w_{\left[v_{1}, v_{2}\right]}=\left|T_{1}\right| /|T|, \quad \int_{\mathbf{s}_{\left((0,1,0,0),\left[v_{1}, v_{2}\right]\right)}} w_{\left[v_{1}, v_{2}\right]}=\left|T_{2}\right| /|T| .
$$

Then

$$
\begin{gathered}
\left|T_{1}\right|=\operatorname{det}\left(\lambda_{v_{T}(i)}\left(v_{T_{1}}(j)\right)\right)=\operatorname{det}\left(\begin{array}{cc}
\lambda_{1}\left(v_{13}\right) & \lambda_{2}\left(v_{13}\right) \\
\lambda_{1}\left(v_{23}\right) & \lambda_{2}\left(v_{23}\right)
\end{array}\right)=\frac{1}{4}|T| \\
\left|T_{2}\right|=\operatorname{det}\left(\lambda_{v_{T}(i)}\left(v_{T_{2}}(j)\right)\right)=\operatorname{det}\left(\begin{array}{cc}
\lambda_{1}\left(v_{1}\right) & \lambda_{2}\left(v_{1}\right) \\
\lambda_{1}\left(v_{12}\right) & \lambda_{2}\left(v_{12}\right)
\end{array}\right)=\frac{1}{2}|T|
\end{gathered}
$$



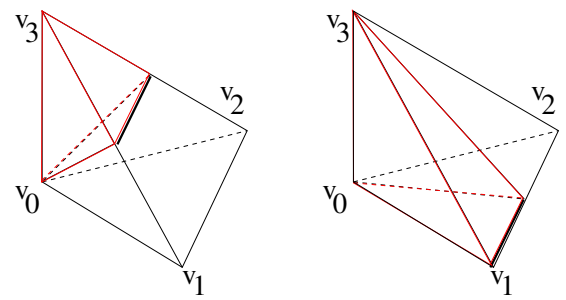

Figure 5: In the tetrahedron $T=\left[v_{0}, v_{1}, v_{2}, v_{3}\right]$, we denote by $v_{i j}$ the mid-point of the edge $\left[v_{i}, v_{j}\right]$. The volume of $T_{1}=\left[v_{0}, v_{13}, v_{23}, v_{3}\right]$ (left), and that of $T_{2}=\left[v_{0}, v_{1}, v_{12}, v_{3}\right]$ (right), both divided by the volume of $T$, give the weights $\left\langle w_{\left[v_{1}, v_{2}\right]}, \mathbf{s}\left((0,0,0,1),\left[v_{1}, v_{2}\right]\right)\right\rangle$, $\left\langle w_{\left[v_{1}, v_{2}\right]}, \mathbf{S}\left((0,1,0,0),\left[v_{1}, v_{2}\right]\right)\right\rangle$, respectively.

where $\lambda_{v_{T}(i)}\left(v_{T_{1}}(j)\right)$ is the $d \times d$ matrix with entries are equal to the value of the barycentric functions associated to the vertices of $T$ (row index $i$ ) at the vertices of $T_{1}$ (column index $j$ ), and similarly for $T_{2}$. Therefore, for $\boldsymbol{\alpha}$ equal either to $(0,0,0,1)$ (left below) or to $(0,0,1,0)$ (right below), we have for example

$$
\int_{\mathbf{s}_{\left((0,0,0,1),\left[v_{1}, v_{2}\right]\right)}} \lambda_{3} w_{\left[v_{1}, v_{2}\right]}=\frac{1}{8}, \quad \int_{\mathbf{s}_{\left((0,1,0,0),\left[v_{1}, v_{2}\right]\right)}} \lambda_{2} w_{\left[v_{1}, v_{2}\right]}=\frac{1}{4} .
$$

Thank to the volume property, it is easy to see that for example

$$
\int_{\mathbf{s}_{\left((0,0,0,1),\left[v_{1}, v_{2}\right]\right)}} w_{\left[v_{0}, v_{2}\right]}=0, \quad \int_{\mathbf{s}_{\left((0,1,0,0),\left[v_{1}, v_{2}\right]\right)}} w_{\left[v_{2}, v_{3}\right]}=0,
$$

since the first is equal to the volume of $\left[v_{13}, v_{23}, v_{2}, v_{3}\right]$ which is zero being $v_{2}, v_{23}, v_{3}$ aligned, and the second is equal to the volume of $\left[v_{0}, v_{1}, v_{1}, v_{12}\right]$ which is zero being two out of four vertices coincident.

Being the small weights unisolvent, another interesting problem is the reconstruction of $z_{h} \in \mathcal{P}_{r+1}^{-} \Lambda^{k}\left(\mathcal{T}_{h}\right)$ in terms of $\left\{\xi_{j}\right\}_{j=1}^{N^{\prime}}$ with assigned weights $w_{i}\left(z_{h}\right)=\int_{\mathbf{s}_{i}} z_{h}$ on the small simplices $\mathbf{s}_{i}$ with $i=1, \ldots, N^{\prime}$. This is clearly related with the interpolation problem on the whole mesh $\mathcal{T}_{h}$ : given $u \in \mathcal{H} \Lambda^{k}(\Omega)$ regular enough to have the weights $w_{i}(u)=\int_{\mathbf{s}_{i}} u$ well defined, we look for $z_{h} \in \mathcal{P}_{r+1}^{-} \Lambda^{k}\left(\mathcal{T}_{h}\right)$ such that $w_{i}\left(z_{h}\right)=w_{i}(u)$ for all $i=1, \ldots, N^{\prime}$.

In this case, the vector $\mathbf{w} \in R^{N^{\prime}}$ with entries $w_{i}\left(z_{h}\right)$ is given and one looks for a solution $\mathbf{Z} \in \mathbb{R}^{N^{\prime}}$ of the singular non symmetric linear system

$$
V_{g} \mathbf{Z}=\mathbf{w}
$$

Then $z_{h}=\sum_{j=1}^{N^{\prime}} \mathrm{Z}_{j} \xi_{j}$.

As we notice before, if a matrix $Q \in \mathbb{R}^{N^{\prime} \times\left(N^{\prime}-N\right)}$ such that its columns are a basis of $\operatorname{Ker}\left(V_{g}^{T}\right)=\left(\operatorname{Im} V_{g}\right)^{\perp}$ is known, then for all $\mathbf{w} \in \mathbb{R}^{N^{\prime}}$ there exists a unique pair $(\mathbf{Z}, \boldsymbol{\Lambda}) \in \mathbb{R}^{N^{\prime}} \times \mathbb{R}^{N^{\prime}-N}$ solution of the linear system (3), namely, such that

$$
\begin{array}{ll}
V_{g} \mathbf{Z}+Q \mathbf{\Lambda} & =\mathbf{w} \\
B \mathbf{Z} & =\mathbf{0}
\end{array}
$$


If $\mathbf{w} \in \operatorname{Im} V_{g}$, then $\boldsymbol{\Lambda}=\mathbf{0}$.

To compute $Q$ one can use the singular value decomposition of $V_{g}=\mathcal{U S V}^{T}$ (see [8]). Matrix $Q$ coincides with the columns from $N+1$ to $N^{\prime}$ of $\mathcal{U}$. Indeed the singular value decomposition is computed in the following way. Since matrix $V_{g}^{T} V_{g}$ is s.s.p. there exists a basis of $\mathbb{R}^{N^{\prime}}$ composed of orthogonal eigenvectors of $V_{g}^{T} V_{g}$ correspondig to the eigevalues $\sigma_{1}^{2} \geq \sigma_{2}^{2} \geq \cdots \geq \sigma_{N}^{2}>0$ (the last $N^{\prime}-N$ eigenvalues are equal zero). The columns for $\mathcal{V}$ are these eigenvectors and the last $N^{\prime}-N$ columns of $\mathcal{V}$ are a basis of $\operatorname{Ker} V_{g}^{T} V_{g}=\operatorname{Ker} V_{g}$. On the other hand the first $N$ columns of $\mathcal{U}$ are equal to $\left(V_{g}[\mathcal{V}]_{i}\right) / \sigma_{i}$, for $i=1, \ldots, N$. These columns are a basis of $\operatorname{Im} V_{g}$. The remaining $N^{\prime}-N$ columns of $\mathcal{U}$ are an orthogonal completion to a basis of $\mathbb{R}^{N^{\prime}}$ so they are an orthogonal basis of $\left(\operatorname{Im} V_{g}\right)^{\perp}$. Notice that solving

$$
\left[\begin{array}{ll}
V_{g} & Q \\
B &
\end{array}\right]\left[\begin{array}{l}
\mathbf{Z} \\
\mathbf{\Lambda}
\end{array}\right]=\left[\begin{array}{l}
\mathbf{w} \\
\mathbf{0}
\end{array}\right]
$$

we compute in fact the best approximate solution of the linear system $V_{g} \mathbf{Z}=\mathbf{w}$ that, using Matlab can be done with the command $\mathrm{Z}=\operatorname{pinv}(\mathrm{Vg}) * \mathrm{w}$.

Remark 5. It is not difficult to select a basis of $\mathcal{P}_{r+1}^{-} \Lambda^{k}$ from the set of generators $\left\{\xi_{i}\right\}_{i=1}^{N^{\prime}}$. The difficult point is the selection of an unisolvent subset of weights. In the case we start from the principal lattice $L_{r}(T)$, we propose to hold the weights corresponding to the indices in the basis, namely $\left\{p_{j}\right\}_{j=1}^{N}$. In all the examples we tested numerically, the generalized Vandermonde matrix $\widetilde{V}_{g} \in \mathbb{R}^{N \times N}$ is invertible.

\section{Conclusions}

In these pages, we have recalled and further commented on the geometrical aspects of the adopted spanning family and set of degrees of freedom (weights) for high order Whitney element spaces on simplicial meshes. Due to the particular generating algorithm, the spanning family requires handling the linear relations which occur among some of its members. A way of counting out these relations is provided (with reference to the so-called "holes") together with the indication about which family members are involved in them (with reference to the boundary of the "holes"). We have also justified theoretically how it is possible to solve a PDE or reconstruct a field from its weights, using the spanning family. Both problems can be reformulated as an algebraic linear system with the same structure.

\section{References}

[1] M. Ainsworth And G. Fu, Bernstein-Bézier bases for tetrahedral finite elements, Comput. Methods Appl. Mech. Engrg., 340 (2018), pp. 178-201. 
[2] D. N. Arnold, Spaces of finite element differential forms, in Analysis and numerics of partial differential equations, vol. 4 of Springer INdAM Ser., Springer, Milan, 2013, pp. 117-140.

[3] D. N. Arnold, R. S. Falk, and R. Winther, Finite element exterior calculus, homological techniques, and applications, Acta Numer., 15 (2006), pp. $1-155$.

[4] M. Benzi, G. H. Golub, And J. Liesen, Numerical solution of saddle point problems, Acta Numer., (2005), pp. 1-137.

[5] M. Bonazzoli, E. Gaburro, V. Dolean, and F. Rapetti, High order edge finite element approximations for the time-harmonic maxwell's equations, CAMA proc., (2014), pp. 1-4.

[6] S. H. Christiansen And K. Hu, Generalized finite element systems for smooth differential forms and stokes' problem, Numer. Math., 140 (2018), pp. $327-371$.

[7] S. H. Christiansen and F. Rapetti, On high order finite element spaces of differential forms, Math. Comp., 85 (2016), pp. 517-548.

[8] G. H. Golub and C. F. Van Loan, Matrix Computations, Johns Hopkins University Press, Baltimore, MD, 1996. Johns Hopkins Studies in the Mathematical Sciences.

[9] J. Gopalakrishnan, L. E. Garcia-Castillo, and L. F. DemkowICZ, Nédélec spaces in affine coordinates, Comp. Math. Appl., 49 (2005), pp. 1285-1294.

[10] J.-C. NÉDÉLEC, Mixed finite elements in $\mathbf{R}^{3}$, Numer. Math., 35 (1980), pp. $315-341$.

[11] F. RAPETti, High order edge elements on simplicial meshes, ESAIM: M2AN, 41 (2007), pp. 1001-1020.

[12] F. Rapetti And A. Bossavit, Geometrical localization of the degrees of freedom for whitney elements of higher order, Special Issue on "Computational Electromagnetism", IET Sci. Meas. Technol., 1 (2007), pp. 63-66.

[13] — Whitney forms of higher degree, SIAM J. Numer. Anal., 47 (2009), pp. 2369-2386.

[14] P. Solin, K. Segeth, And I. Dolezel, High-order finite element methods, Chapman \& Hall/CRC, 2004. Studies in Advanced Mathematics.

[15] J. Xin, N. GuO, AND W. CAI, On the construction of well-conditioned hierarchical bases for tetrahedral h(curl)-conforming nédélec element, J. Comp. Math., 29 (2011), pp. 526-542. 